\title{
Chondrule-like objects formed by arc discharges aboard the ISS
}

T. E. $\mathrm{KOCH}^{1 *}$, D. SPAHR ${ }^{1}$, D. Merges' ${ }^{1}$, A. A. BeCK ${ }^{1}$, O. Christ $^{1}$, S. Fujita ${ }^{1}$, P.-T. GenzeL ${ }^{1}$, J. KERSCHER ${ }^{1}$, M. LINDNER ${ }^{1}$, D. MEDEROS LEBER ${ }^{2}$, B. WINKLER ${ }^{1}$, F. E. BRENKER ${ }^{1}$.

${ }^{1}$ Goethe University Frankfurt, Institute of Geoscience, Altenhoeferallee 1, 60438 Frankfurt am Main, Germany ${ }^{2}$ Goethe University Frankfurt, Department of Physics, Maxvon-Laue-Strasse 1, 60438 Frankfurt am Main, Germany (*correspondence: t.koch@em.uni-frankfurt.de)

Chondrule formation theories are still controversially debated and remain among the most discussed issues in studies concerned with planet formation. The formation of chondrules by nebular lightning belongs to one of the most plausible scenarios [1-5]. We developed an experiment named EXCISS (Experimental Chondrule Formation aboard the International Space Station (ISS)) which was carried out in micro gravity aboard the ISS. In this experiment, synthetic forsterite $\left(\mathrm{Mg}_{2} \mathrm{SiO}_{4}\right)$ dust particles which were levitating in a glass sample chamber were exposed to arc discharges. The aim of these experiments was to explore if dust particles melt, fuse and/or form chondrule-like objects with the help of arc discharges [6,7].

The experiment returned in August 2019. More than 80 objects which are clearly distinct from the initial sample material could be identified. These objects vary from small droplet shaped entities to aggregates of more than 10 particles which were analyzed by various techniques. The results clearly show that in our experiment aboard the ISS we were able to completely melt $100 \mu \mathrm{m}$ sized particles and fuse several initial grains into aggregates. Notably, melting induced by arc discharges was not only restricted to grain surfaces.

References: [1] Whipple (1966) Science 153, 54-56. [2] Horányi et al. (1995) Icarus 114, 174-185. [3] Desch \& Cuzzi (2000) Icarus 143, 87-105 [4] Johansen \& Okuzumi (2018) Astronomy \& Astrophysics A31, 1-22 [5] Güttler et al. (2008) Icarus, 195, 504-510 [6] Koch et al. (2018) Ann. Meet. Met. Soc. Abstract \#6139 [7] Koch et al. (2019) LPSC $\# 1560$

Acknowledgments: The experiment is supported by the German Aerospace Center DLR, DreamUp, NanoRacks LLC, the Dr. Rolf M. Schwiete Stiftung, the DFG (BR2015 / 35-1) and the BMWi (50JR1704), NORDLICHT GmbH, BIOVIA and ZEISS. A special thanks goes to the Hackerspace Ffm e.V.. 